\title{
NUEVA FORMA ALTERNATIVA DE SOLUCION DE CONTROVERSIAS EN EL AMBITO DEL MERCOSUR: CYBERTRIBUNAL DEL MERCOSUR
}

Ricardo Ignacio Beltramino Herren*

SUMÁRIO; 1. Introdução. 2. Delineamento do Problema. 3. Antecedentes. 4. Serviços oferecidos pelo Cybertribunal do MERCOSUL. 5. Financiamento. 6. Conclusão. 7. Bibliografia.

SUMMARY: 1. Introduction. 2. Problem Delineation. 3. Antecedents. 4. Services offerd for MERCOSUL Cybertibunal. 5. Financing. 6. Conchusion. 7. Bibliography.

SUMARIO; 1. Introducción, 2. Planteamiento del Problema. 3. Antecedentes. 4. Servicios que ofrece el Cybertribunal del MERCOSUR. 5. Financiamento. 6. Conciusión. 7. Bibliografia.

RESUMO: O objeto deste trabalho é delinear a conveniência de instrumentalizar un sistema que permita aos particulares encontrar vias alternativas de solução de controversias.

ABSTRACT: The object of this work is to delineate the convenience to create a system allows the particular ones to find alternative ways of dispute settlement.

RESUMEN: El objeto de este trabajo es plantear la conveniencia de la instrumentación de un sistema que permita a los particulares encontrar vias alternativas de solución de controversias.

PALAVRAS-CHAVE: Mercosul. Cybertribunal. Solução de controvérsias.

KEY-WORDS: Mercosul. Cybertribunal. Dispute settlement.

PALABRAS-LLAVE: Mercosur. Cybertribunal. Solucion de controversias. 


\section{Introducción}

El proceso de integración regional en el que se enmarca el Mercosur tiene hoy una crisis de crecimiento. La voluntad política de los presidentes de cada uno de los países y la fuerza del comercio han hecho posibles numerosos logros como lo es el notable crecimiento del intercambio comercial principalmente entre Argentina y Brasil.

Y es precisamente entre estos dos países donde se han suscitado, en el transcurso de 1999 y 2000 una gran cantidad de conflictos sectoriales que produjeron tensiones en las relaciones políticas internacionales de ambos países.

Hoy vemos con preocupación que el avance del proceso integrador peligra ya que los instrumentos previstos para la solución de controversias dentro del bloque regional no poseen la celeridad y eficacia que estas requieren.

Además, los particulares se encuentran alejados de la participación ya sea de los reclamos como de las defensas frente a estos, lo que trae aparejada mucha incertidumbre y desconfianza entre la población acerca no sólo de la resolución del conflicto sino también de la necesidad de un proceso de integración regional.

Resulta imprescindible que los habitantes de los países miembros del bloque entiendan la conveniencia del proceso integrador y que encuentren en el mismo soluciones concretas a sus necesidades.

El objeto de este trabajo es plantear la conveniencia de la instrumentación de un sistema que permita a los particulares encontrar vías alternativas de solución de controversias.

\section{Planteamiento del problema}

a) Algunos autores como Andorno, sostienen la conveniencia de crear un Tribunal supranacional que se encargue de la solución de diferencias entre los países miembros del Mercosur. Sin embargo, hoy la conformación de órganos supranacionales es vista con recelo, especialmente por Brasil ya que los perciben como una cesión de soberanía. Además, la estructura administrativa del Mercosur ya existente, lamentablemente no ha demostrado una funcionalidad óptima, por lo que crear un sistema burocrático supranacional con esos antecedentes 
no nos garantiza efectividad, y los costos que implicaría su mantenimiento también constituirían otra traba. Por eso pensamos que tratar el tema en este estadio de la integración no parece por lo menos oportuno.

b) Por su parte, los procedimientos contra las prácticas que afectan a la industria establecidos en el ámbito administrativo de los países miembros, como los previstos por la Subsecretaría de Comercio Exterior y la Comisión Nacional de Comercio Exterior en el caso de Argentina, y su similar en Brasil, están sujetos a las normas del GATT 94, el Entendimiento sobre solución de diferencias y sus Acuerdos complementarios como el relativo al dumping, acuerdo sobre salvaguardias y el acuerdo sobre subvenciones y medidas compensatorias.

Estos mecanismos tienen exigencias de procedimiento y de investigación que no siempre están al alcance de los "damnificados" por el daño grave o la amenaza de daño al sector al que pertenecen. A su vez, el comercio internacional se mueve con una rapidez que contrasta con los tiempos requeridos por la administración pública para mover sus engranajes.

Al mismo tiempo las normas y la percepción comunitarias chocan con las reglas establecidas en los Acuerdos de la OMC. El caso de las salvaguardias resulta ejemplificador; por un lado la O.M.C. exige que las salvaguardias se apliquen a las importaciones de todos los estados miembros sin exclusiones regionales, así en el caso del conflicto suscitado en relación con las salvaguardias impuestas por la Argentina en materia de calzados el Organo de Apelación de la OMC llega a la conclusión de que " la investigación realizada por la Argentina en el caso presente no puede servir como base para excluir a las importaciones procedentes de otros Estados miembros del MERCOSUR de la aplicación de las medidas de salvaguardia."

Y por el otro, no tiene dentro del marco del Mercosur instrumentos que le permitan aplicar salvaguardias contra otros miembros de la Unión Aduanera. Así lo ha entendido, si bien en el caso particular, el Tribunal Arbitral en su tercer laudo sobre las salvaguardias argentinas impuestas en materia de textiles.

c) El hecho de que el ámbito judicial de cada país no está acostumbrado a la aplicación de normas de derecho internacional privado y muchas veces no posee la infraestructura para que la solución de las diferencias sean lo rápidas que exige el comercio agrava el panorama.

\footnotetext{
' Organo de Apelación de la O.M.C. Decision de fecha 17 de noviembre de 1999 y publicadá el 19 de diciembre de ese año
} 
Claros han sido los ejemplos en casos planteados ante la justicia laboral argentina en donde la dilación producida por el desconocimiento por parte de los Tribunales de los pasos a seguir así como la necesidad de que los trámites se realicen a través de Cancillería demora el proceso de una manera notable. Asimismo los fallos dictados por la Cámara Federal de la provincia de Entre Ríos (Argentina), estableciendo cupos de importación para el ingreso de pollos de Brasil, y por un Tribunal Regional Federal de la $4^{\circ}$ Región en Rio Grande do Sul (Brasil) limitando el ingreso de arroz argentino (aunque luego revocada por el Superior Tribunal de Justicia) corroboran por un lado el hecho de que los particulares no obtienen soluciones adecuadas del poder ejecutivo y al mismo tiempo, que el sistema jurídico se arroga competencias frente al comprensible clamor de los damnificados.

En coincidencia con lo expuesto el lunes 13 de marzo de 2000 la ex Secretaria de Industria de Argentina Débora Giorgi declaró que:

"El actual marco institucional es insuficiente. No contempla mecanismos para evitar el incumplimiento de los compromisos acordados ni tampoco instancias efectivas para manejar los conflictos que surgen entre los socios. Las tensiones del último año ponen en evidencia estas falencias. Pensamos que es imprescindible el desarrollo institucional del Mercosur de manera de asegurar la internalización de las normas pactadas y fortalecer el mecanismo de solución de controversias".?

d) La constitución Tribunales arbitrales ad hoc propuestas por el Protocolo para la Solución de Controversias conocido como el Protocolo de Brasilia ha demostrado que su utilización no es frecuente (a la fecha de este trabajo sólo se conocen tres laudos), y presenta la dificultad de que los particulares no tienen una intervención directa en la resolución de los conflictos ya que la controversia tiene como sujetos únicamente a los Estados partes.

El objeto de estas líneas es proponer una alternativa para la resolución de disputas utilizando instrumentos operativos como lo son el Tribunal Arbitral de C.O.A.D.E.M. (Colegios y Ordenes de Abogados del Mercosur) e INTERNET. Nos estamos refiriendo al CYBERTRIBUNAL DEL. MERCOSUR 


\section{Antecedentes}

a. C.O.A.D.E.M. es una institución que nuclea a la abogacía organizada del Mercosur, y está integrada por los Colegios y Ordenes de abogados de los países integrantes del bloque regional representando a más de 500.000 abogados. La función social de la abogacía y su necesaria presencia en la resolución de los conflictos sean estos entre particulares o entre Estados colocan a esta institución en una posición de privilegio ya que además de no constituir un órgano burocrático (ya que sus miembros son delegados de cada uno de los Colegios y Ordenes independientes), la diversidad geográfica de su estructura y la cualificación de sus miembros facilita la instrumentación del proyecto.

b. C.O.A.D.E.M. tiene aprobado la conformación de un Tribunal Arbitral. En este acuerdo se establecen ámbitos de incumbencia, nombramiento de árbitros, etc., y ha sido presentado ante las autoridades de los respectivos países y las autoridades administrativas del MERCOSUR.

Sin embargo, hasta el momento, las dificultades y costos de su estructura a los que se le suma la falta de práctica, hacen que no se hayan presentado disputas a su conocimiento.

c. Internet se ha transformado en los últimos años en una herramienta de comunicación excepcional y lo será sin dudas en un futuro no muy lejano cuando el e commerce adquiera en nuestros paises la dimensión que tienen en los países centrales.

Resulta ser el medio más propicio para acercar a las partes de países como los nuestros en los que la distancia puede constituirse en una barrera.

Existen materias como los relacionados con el Comercio internacional, competencia desleal, derechos de marcas y patentes, e-commerce, entre otros, excluyendo los que se encuentran bajo la órbita del derecho criminal que pueden ser utilizadas CYBERTRIBUNAI DEL MERCOSUR.

c. Páginas relacionadas con resolución de controversias por Internet:

Hoy existen numerosos sitios en la red Internet que abordan la posibilidad de resolver los conflictos vía Internet. Generalmente se relacionan con problemas generados por el uso de la red, ya sea nombres de dominio, privacidad, e commerce, cuestiones contractuales .

A continuación una lista de algunos de estos y sus responsables: 
www.clicknsettle.com www.Cybersettle.com www.eresolution.ca www.i_courthouse.com www.courtcity.com www.mediate.com www.arbiter.wipo.int/center/index-es.html www.settlementnow.com Ray William London PhD http:/www.LondonMedArb.com Dan \& Heidi Chay http:/www.learning-communities.com Gerald Goulder www.zerolitigation.com Lewis Shadoff http://www.ResolutionForum.org Frost Branon http://www.branon.com Julie Denny www.resolutionsforyou.com Nora Femenia www.inter-mediacion.com David Southern www.camosun.bc.ca/divisions/ombud David Larson http:/web.hamline.edu/law/adr Ernest Thiessen www.oneaccordinc.com Tonia Brown http:/www.mediation-omc.org Anthony Kreindler www.adrworld.com Jim Melamed http:/www.to-agree.com Catherine Morris www.peacemakers.ca Mary Lou Addor www.ces.ncsu.edu/PIE/nrli Jodie Lanzisera http:/www.findlaw,com John Stephens http:/ ncinfo.iog.unc.edu/programs/dispute/index.html Linda Baron www.nafcm.org

Eben Weitzman http://www.umb.edu/disres

Ann Zenk www.lawyeriowa.com

Martin Smith www.mediate.com/martinsmith

Helie John R. www.mediate.com/jrhelie

Lisa Cameron www.ombuds.net

Dan Dozier www.tlisystems.com

Kristina Eisenacher www.keylaw.com

Tammy Lenski http:/ www.lenski.com

Tower Guy http:/www.kaufmanandcanoles.com/

AttorneyDetail.asp?AtCode106\&sEmail=gktower@kaufcan.com Adam Gersch www.globalmediation.co.uk 
Archie Zariski http://carmen.murdoch.edu.au/ zariski

John Ford http:/www.mediate.com/johnford/

Ronald Surratt http:/ www.harddecisions.com

Charles Crumpton http:/www.paclawteam.com

Hon. Alice D. Sullivan (Ret.) www.Privateludge.com

Frederic Conway www.va.gov/adr

Derek Sweetman www trinstitute.org

linda hack http://rampages.onramp.net/-herlaw

Jeff Citrin www.resolv.org

Larry Whitted www.mediate.com/lwhitted

Kay Min http://ceo.cudenver.edu/ kyung_min/

Robert Wildau www.talkworks.com

Gina Boltz www.turtle-tracks-for-kids.org

Ken Bryant www.mediate.com/California

Desmond M. Connor www.connor.bc.ca/connor

Sanford Lewis

http:/home.earthlink.net/ gnproject/strategiccounsel.htm

Tom Buckner http:/www.courts.state.ny.us/adr

Jennifer Tucker http:/www.concentric.net/ tuckerj

Ken Shigley http:/atlantainjurylawyer.com

Tobie Wolf www.adro.net

Tobie Wolf www.adro.net

Edward Burton http:/ www.consultburton.com

Tom Taylor http:/consensus.fsu.edu

Dr. Michael Hynes memberexpertpages.com/aviaiton expert

Dr. Michael Hynes member.expertpages.com/aviaiton_expert

Singh Lisa http:/ www.dnaco.net/ lisa

Matt Johnson www.masonatlas.com

Jeffrey Krivis www.firstmediation.com

Eduardo Alberto Marfany Lastra http:/www.mediacion.com

Robert Price www.enteract.com/ bobprice/

Sallyann Roth www.publicconversations.org

Yasutaka Machimura http:/www.asia-u.ac.jp/ matimura/

Rothman Jay www.aepro.org and www.ariagroup.com

Christina Kallas WWW.LAWYERS.COM/KALLASLAW

Charles Mudd http:/www.mudd.org

Nancy K. Ferrell www.flash.net/ nferrell 
James L. Stovall, Jr http:/ www.mediationinstitute.com

Evelyn Gaites www.ogc.secnav.hq.navy.mil/adr

Thomas Repicky www.Lawyers.com/thomasrepicky

Ronnie Wilson http:/ www.atsdr1.atsdr.cdc.gov:8080

Hank Moorlag ombudsman.yk.ca

Susan Patlyek www.chss.montclair.edu/leclair/LS/msu2.html

Ron Myers www.ronmyers.com

Margaret Nichols http:/home.att.net/ rmnichols/index.html

Michael Chapman www.interarb.com/vl/pages/

Wayne Smith www.wesleyan.net/wsmith.html

Jeanette G. Smith www.demurrage.com

Natalie Fleury www.demarsassociates,com

Christine Coates mediationnow.com/coates/index.html

Paulette Kohman http:/firms.findlaw.com/paulette/index.htm

Anthony Palladino www.faa.gov/agc www.adr.af.mil/iadrwg/

Clyde Long www.i-courthouse.com

Clyde Long www.i-courthouse.com

Chris Brien http:/www.csu.edu.au/cyber

Michael Wolf home.earthlink.net/mjwolfsa/hotlinks

Alexandria Wudyka www.brudder.com

Grover Partee www.ursusconsulting.com

John Horn www.ADRInternational.com

Marc Miller www.solutionsmediation.com

Kenneth Frank http:/www.brenau.edu/humanities/frank/

J.K. Miller http:/www.ericksonmediation.com

Maggie Kennedy www.consensus.uk.com

Mary Hillebrand http://www.specialednews.com

van Welsem Fred W. www.welsem.nl

Fred W. van Welsem www.welsem.nl

Rosa Rios www.wonderrose.freelife.com

Laina Reynolds http:/www.brad.ac.uk/acad/confres/

Daniel Wax www.SolutionsMediation.com

Verner Smitheram www.upei.ca/ conflictcentre

Mary Wright www.manatt.com

Richard Schnoll www.cybersettle.com

Nanette Castle www.cdsadr.org

Mike Niemeyer WWW,doj.state.or.us/ADR 


\section{Giancarlo Francini http://members.tripod.it/itfs/}

Patricia Paul http://firms.findlaw.com/PatriciaPaul/

Sharon Ralph www.ocsea.org/obes-assembly

James Foskett www.theiapn.com or www.webdisputeresolutions.com Douglas Yarn law.gsu.edu/dyarn/

Jo Dee Davis http://www.peace-center.org

Dr Loukas Mistelis http://www.ccls.edu/eclu

JOHN HUTTON www. Vcu.edu/busweb/vlsc

Donald Lloyd www.Diversifiedconsulting.net

Lerman Edie www.rent-a-court.com

Colin Rule www.onlinemediators.com

Roger Pitchforth http://www.massey.ac.nz/ wwdisre/

El común denominador es la utilización de formatos básicos como lo es el sistema EDI Electronic Data Interchange (INTERCAMBIO ELECTRONICO DE DATOS). . Este es definido como: "El intercambio automatizado de documentación normalizada en formularios electrónicos, que se realiza directamente desde las aplicaciones de un computador hacia las aplicaciones de otro u otros computadores, en relaciones tipo empresa-empresa, empresa-administración"

O como lo señala la Ley Modelo CNUDMI en su ART. 2 b) Por "intercambio electrónico de datos (EDI)" se entenderá la transmisión electrónica de información de una computadora a otra, estando estructurada la información conforme a alguna norma técnica convenida al efecto;"

Es decir que mediante este sistema se pueden transmitir formularios preestablecidos en donde las partes pueden completarlo con sus demandas, contestaciones, pruebasy alegatos así como la resolución final que adopte el Tribunal.

\section{Servicios que offece el CyberTribunal del MERCOSUR}

1) El Tribunal tendría una " sede virtual " es decir una página web en la red INTERNET posibilitando que tanto los árbitros como los representantes de las partes se encuentren en sus respectivos países, lo que abarataría los costos. En los supuestos que fuera necesario la presencia de testigos o documentación como prueba del proceso se podrían 
presentar o acompañar en el Colegio de Abogados de la jurisdicción respectiva en donde se podrían tomar las declaraciones y en donde se certificaria la autenticidad de los documentos acompañados.

2) El Cybertribunal del MERCOSUR ofrecería a las personas que tengan una disputa, primero un proceso de mediación.

1. En este proceso en el que dos personas plantean sus puntos de vista el Mediador no tiene el poder de imponer o entregar la solución. Simplemente propone una solución en forma de compromiso después de evaluar las partes y sus puntos de vista, trabajando junto a las partes en identificar sus discordancias y teniendo en cuenta sus intereses.

El Mediador orienta la discusión para que las partes lleguen a un compromiso haciendo notar que siempre están en una etapa de mediación.

Pensamos que este puede ser el instrumento muy útil para la resolución de conflictos sectoriales.

2. La otra posibilidad es el arbitraje, definido como un proceso en el que dos partes presentan su conflicto a un tercero neutral, el arbitro. El proceso es más informal que en los tribunales normales y sus decisiones son inapelables.

3. Este Tribunal tiene como ventaja por un lado su confidencialidad a través de un sistema de encritpado al que sólo tienen acceso las partes.

4 Estarian facultados para actuar unicamente abogados matriculados en los Colegios de Abogados.

5. Cada representado retribuiría a su profesional.

\section{Financiamiento}

Entre las múltiples posibilidades de conseguir financiamiento destacamos la que se podría conseguir encontramos:

a. El cobro de honorarios por los servicios prestados a las partes en disputa.

b. El cobro de una suma fija mensual a los Colegios de Abogados

c. La financiación a través de los fondos de Cooperación internacional que disponen las cancillerías.

d. El auspicio de fundaciones internacionales como la Hewlett 
Foundation. Esta es una fundación con sede en estados Unidos que financia programas tanto en el área de las relaciones de Estados Unidos con Latinoamérica como en el área de Resolución de Conflictos.

Los objetivos del "US-latin American Relations Program" están basados en tres premisas:

"1) Continuar con la integración hemisférica es inevitable y representa un formidable desafio para California y los Estados Unidos.

2) La forma que esa integración tome en las Américas puede ser positivamente influenciada con la inversión a tiempo en instituciones, recursos humanos e infraestructura,

3) El principal obstáculo para el beneficio mutuo en las relaciones está en la falta de contacto, colaboración y cooperación entre las instituciones y los individuos y la idea equivocada, desconfianza pérdida de respeto y pronunciado desequilibrio en el poder que caracterizan las relaciones en el hemisferio “

El programa se enfoca a proveer apoyo que sirva a incrementar la capacidad institucional. Y dentro de este apoyo está el referido "especialmente a asociaciones profesionales "y a aquellas iniciativas que conduzcan actividades en colaboración con instituciones asociadas.

Encontrándose como regiones prioritarias para el proyecto "Chile, Argentina y Brasil".

Dentro de las instituciones asociadas se encuentra la Universidad de Massachusett que cuenta con un interesante proyecto de difusión de las posibilidades que ofrece Internet para el derecho, y especialmente la resolución de controversias en dicho ámbito.

Siendo otro de los objetivos a apoyar por la Hewlett Foundation el de la Resolución de Conflictos sería coincidente el área promocionada.

En efecto, la Hewllet Foundation apoya a un limitado número de organizaciones que están trabajando en la aplicación internacional de la resolución de conflictos, técnicas y desarrollo de prácticas relacionadas con conflictos étnicos, ideológicos, raciales y otros grupos de conflicto, siendo esta la única categoría dentro del Programa de Resolución de Conflictos que son consideradas iniciativas internacionales. 


\section{Conclusión}

Por los motivos expuestos o estimo que sería conveniente para C.O.A.D.E.M. la implementación de un CYBERTRIBUNAL DEL MERCOSUR que podria agilizar la tarea de solucionar diferencias y ser una fuente genuina de ingresos que permita su constante crecimiento.

Estas son sólo algunas ideas que sometemos a su consideración y que consideramos pueden contribuir al desarrollo del proceso integrador y brindar al mismo tiempo un nuevo ámbito de actuación profesional en el MERCOSUR.

\section{BIBLIOGRAFÍA}

MERCOSUR hay que nivelar los incentivos. Diário El Cronista Comercial. $13 / 03 / 00$. p. 4 y 5 . 